Revue internationale P.M.E.

Économie et gestion de la petite et moyenne entreprise

\title{
Facteurs d'adoption des nouvelles technologies de production dans les PME manufacturières
}

\section{Elisabeth Lefebvre, Louis-A. Lefebvre et Dominique Colin}

Volume 3, numéro 2, 1990

URI : https://id.erudit.org/iderudit/1007978ar

DOI : https://doi.org/10.7202/1007978ar

Aller au sommaire du numéro

Éditeur(s)

Presses de l’Université du Québec

ISSN

0776-5436 (imprimé)

1918-9699 (numérique)

Découvrir la revue

Citer cet article

Lefebvre, E., Lefebvre, L.-A. \& Colin, D. (1990). Facteurs d'adoption des nouvelles technologies de production dans les PME manufacturières. Revue internationale P.M.E., 3(2), 215-229. https://doi.org/10.7202/1007978ar
Résumé de l'article

L'étude de l'adoption des technologies avancées de production auprès d'un échantillon de PME manufacturières innovatrices a permis d'identifier six facteurs principaux. Ces six facteurs représentent différentes dimensions liées aux influences externes et internes de l'entreprise, aux caractéristiques organisationnelles et financières, et aux stratégies poursuivies lors de l'adoption. L'étude comparative du classement de ces facteurs dans deux groupes d'entreprises de degré innovateur différent suggère l'existence d'un modèle évolutif d'adoption. 


\title{
Facteurs d'adoption des nouvelles technologies de production dans les PME manufacturières innovatrices
}

\author{
Élisabeth LEFEBVRE* \\ Université du Québec à Montréal \\ Louis-A. LEFEBVRE* \\ Université du Québec à Montréal \\ Dominique COLIN** \\ Université du Québec à Montréal
}

\begin{abstract}
RÉSUMÉ
L'étude de l'adoption des technologies avancées de production auprès d'un échantillon de PME manufacturières innovatrices a permis d'identifier six facteurs principaux. Ces six facteurs représentent différentes dimensions liées aux influences externes et internes de l'entreprise, aux caractéristiques organisationnelles et financières, et aux stratégies poursuivies lors de l'adoption. L'étude comparative du classement de ces facteurs dans deux groupes d'entreprises de degré innovateur différent suggère l'existence d'un modèle évolutif d'adoption.
\end{abstract}

* Elisabeth Lefebvre est professeur agrégé au Département des sciences comptables. Louis A. Lefebvre est professeur titulaire au Département des sciences administratives et membre du CREDIT (Centre de recherche en développement industriel et technologique). Dominique Colin était chercheur au Centre de recherche en gestion. Leur adresse est: C.R.G., UQAM, C.P. 8888, Succursale A, Montréal, Québec, H3C 3P8. Premier manuscrit reçu en février 1990.

** Recherche subventionnée partiellement par un fonds CRSH (W 312 L2315).

Les auteurs voudraient remercier les trois lecteurs anonymes pour leur suggestions et commentaires qui ont permis d'améliorer sensiblement la version finale de ce texte, ainsi que Sylvie Robidoux et Carl St-Pierre pour leur assistance de recherche.

Cet article est dédié à la mémoire de notre collègue et amie Dominique Colin, décédée avant la parution de ce numéro de la revue. 


\begin{abstract}
In a study of the determinants of adoption carried out in a sample of small innovative manufacturing firms, six main factors associated with adoption were identified. These factors represented different dimensions related to the characteristics of the organizations, the strategies pursued by the firms, and the internal and external influences on the acquisition decision. A comparative analysis of two groups of firms of differing levels of automation indicated that factors of adoption were different suggesting that adoption follows an evolutionary pattern.
\end{abstract}

\title{
RESUMEN
}

Un estudio realizado por medio una muestra de pequeñas y medianas impresas manufactureras innovadoras, con respecto a la adopción de tecnologias avanzadas de producción, ha permitido indentificar seis factores principales. Esos seis factores representan diferentes dimensiones ligadas a las influencias internas y externas de la empresa, a las caracteristicas organizacionales y financieras, y a las estrategias seguidas durante dicha adopción. El estudio comparativo de los factores, en dos grupos de firmas de distintos niveles de automatización, sugieren que las diferencias notadas se deben al hecho de que la adopción sigue un modelo evolutivo.

Mots clés : Facteurs d'adoption, technologies avancées de production, PME innovatrices 


\section{1}

\section{Introduction}

Il est de plus en plus reconnu que l'adoption des nouvelles technologies représente une source importante d'avantages concurrentiels pour les entreprises manufacturières (Thurow, 1987; NRC, 1985). Cette constatation revêt une importance toute particulière dans le contexte actuel d'ouverture commerciale et de globalisation des marchés. Or, plusieurs auteurs dénoncent la lenteur des entreprises manufacturières nord-américaines à adopter les nouvelles technologies de production (Litvack et Warner, 1987; McNeil et Dilon, 1987; Thurow, 1987) avec, comme conséquence, un secteur manufacturier de moins en moins compétitif sur le plan international. Bien que l'Amérique demeure un leader dans sa capacité à générer l'innovation technologique et à consommer les produits qui en découlent, elle est cependant déficiente au niveau de l'adoption de l'innovation à des fins de production et de fabrication (Gomory, 1989; Thurow, 1987). L'innovation au niveau des procédés constituerait donc à cet égard le principal défi de la prochaine décennie (Lefebvre et Lefebvre, 1988).

Pour la PME, ce défi, en plus d'en être un de taille, pourrait également constituer un enjeu vital. En effet, les petites et moyennes entreprises seraient plus vulnérables aux pressions compétitives engendrées par la globalisation croissante des marchés (CEC, 1988). Cette vulnérabilité proviendrait non seulement d'une carence en ressources, notamment en personnel qualifié et en capitaux, mais aussi du degré peu élevé de sophistication technologique de ces entreprises. Cependant, l'adoption des nouvelles technologies de production n'apparaît pas exclue pour les plus petites entreprises. Plusieurs recherches récentes démontrent que les petites entreprises possèdent certains avantages qui facilitent l'adoption de ces nouvelles technologies (Meredith, 1987). Les petites entreprises souffriraient moins d'inertie organisationnelle et, par conséquent, seraient plus aptes à répondre rapidement aux pressions internes et externes. De plus, la participation généralement plus grande des employés aux activités de l'entreprise favoriserait les changements organisationnels dont ceux engendrés par l'adoption et la mise en place des nouvelles technologies. Enfin, les petites entreprises seraient plus en mesure d'exploiter certains des avantages concurrentiels que procure l'adoption des nouvelles technologies telles que la flexibilité accrue du système de production, les plus grandes variétés et spécificités des produits offerts, ainsi qu'un meilleur service à la clientèle.

Peu de PME ont adopté des technologies avancées de production bien que certaines d'entre elles, plus innovatrices, aient accepté de relever ce défi. Il est proposé dans le cadre de cet article d'étudier empiriquement les principaux facteurs d'adoption des nouvelles technologies de production dans un groupe de ces entreprises. 


\section{Les déterminants d'adoption des nouvelles technologies}

L'adoption des nouvelles technologies à base informatique constitue une forme d' «innovation au niveau des procédés» (méthodes de gestion et de production) par opposition à «innovation au niveau des produits» (création de nouveaux produits dont certaines caractéristiques sont différentes des produits précédents). Cette distinction classique a été proposée par plusieurs auteurs (par exemple, Pennings, 1987 ou Hill, 1979). L'innovation au niveau des procédés peut, à son tour, inciter d'autres formes d'innovations organisationnelles compte tenu de l'effet de synergie généré par le changement. Bien que la recherche sur l'innovation ait soulevé beaucoup d'intérêt (dès 1975, Rogers et Eveland ont relevé plus de 2400 études sur le sujet), un consensus se dégage selon lequel l'état des connaissances sur les déterminants d'adoption d'innovations demeure très restreint (Meyer et Goes, 1987; Pennings, 1987). Ceci est d'autant plusévident lorsque le cas précis de l'adoption des technologies avancées de production est considéré : bien que certains cadres conceptuels aient été proposés (par exemple, Gasse, 1989; Farley et al., 1987), peu de recherches empiriques semblent exister. Quelques exceptions connues sont, entre autres, les recherches de Farley et al. (1987) et Dean (1987) effectuées sur les grandes entreprises manufacturières, et celles de Julien et al. (1988) menées dans le cadre de plus petites entreprises.

L'analyse des recherches antérieures en innovation permet d'identifier un certain nombre de déterminants susceptibles de promouvoir l'adoption des innovations dans une organisation. Nous en avons retenu quatre ensembles pour cette recherche :

i) les déterminants associés aux caractéristiques des organisations étudiées, telles que la taille (Meredith, 1987; Farley et al., 1987), la performance financière (Farley et al., 1987; Clark et al., 1985), et l'effort innovateur (Ettlie et Bridges, 1987, 1982), plus particulièrement au niveau des ressources consenties à la recherche et au développement (Robertson et Gatignon, 1987; Kamien et Schwartz, 1982);

ii) les déterminants associés à la stratégie organisationnelle (Meyer et Goes, 1987; Ettlie et Bridges, 1987) et à la stratégie technologique de l'entreprise (Ettlie et Bridges, 1987; Child, 1987; Clark et Hayes, 1987; Hayes et Wheelwright, 1984); le maintien et la poursuite de stratégies concurrentielles représenteraient aussi des éléments cruciaux lors de l'adoption puisqu'elles sont étroitement liées à l'utilisation, par exemple, de l'ensemble des nouvelles technologies de l'information et de production (Quinn et al., 1990; Porter et Millar, 1985; Parsons, 1983) et ce, même dans le cas spécifique des entreprises de plus petite taille (Bergeron et Buteau, 1988; Meredith, 1987);

iii) les déterminants associés au processus décisionnel (Dean, 1987; Pennings, 1987; Meyer et Goes, 1987), en particulier l'influence du dirigeant (Julien et al., 1988; Miller et Toulouse, 1986), l'influence du groupe ingénierie-production 
(Lefebvre et Lefebvre, 1990; Collins et al., 1988; Ettlie et al. 1984), l'influence du groupe marketing (Ettlie et Bridges, 1987; NRC, 1985), l'influence des fournisseurs de la technologie et des consultants externes (Weiss et Birnbaum, 1989; Robertson et Gatignon, 1987), et l'influence des clients (Weisset Birnbaum, 1989; Meredith, 1987; Ettlie et Bridges, 1982);

iv) les déterminants associés aux caractéristiques des innovations adoptées tels le coût et le degré de fiabilité (Julien et al., 1988; Meyer et Goes, 1988; Pennings, 1987).

Notons que les déterminants retenus ici ne s'inscrivent pas dans une perspective macro (sectorielle ou nationale) comme celle privilégiée par Naisbett (1982), mais dans une perspective micro (niveau de la firme) telle que définie par Abernathy et al. en 1981 et reprise par Hayes et Wheelwright en 1984. Selon cette perspective, l'accent porte essentiellement sur des considérations organisationnelles et managériales. Au total, 18 variables ont été retenues et sont présentées dans la figure 1 (p. 220).

Un double objectif est poursuivi dans cette recherche : d'une part, explorer les interrelations entre variables et regrouper ces dernières en un nombre plus restreint de facteurs correspondant aux différentes dimensions associées à l'adoption des technologies avancées de production dans les PME innovatrices; d'autre part, hiérarchiser ces dimensions en tenant compte de deux groupes d'entreprises innovatrices de degrés innovateurs différents.

\section{$3 \quad$ Méthodologie}

Cette recherche a été menée auprès d'un échantillon systématique de toutes les entreprises manufacturières répertoriées dans le fichier central des entreprises du Québec. L'enquête postale par questionnaire a été choisie pour la collecte des données; le questionnaire était adressé aux dirigeants des entreprises. Ce choix de répondant a été dicté en raison de la connaissance que ce dernier possède de son entreprise en général, de l'influence qu'il exerce dans la direction stratégique de son entreprise (Miller et Toulouse, 1986), et du rôle qu'il joue lors de l'adoption des nouvelles technologies (Julien et al., 1988; Skinner, 1985). Les dirigeants de 668 entreprises ont retourné un questionnaire dûment rempli et, de ce nombre, 651 questionnaires validés ont été retenus.

Les entreprises sélectionnées pour les fins de cette recherche étaient des entreprises manufacturières de moins de 200 employés ayant adopté au moins une des technologies avancées de production suivantes : la conception assistée par ordinateur, la fabrication assistée par ordinateur, les commandes numériques informatisées, la 'manutention automatisée, l'inspection et le contrôle de la qualité 
Figure 1

Varlables de recherche, type de mesures et justification théorique

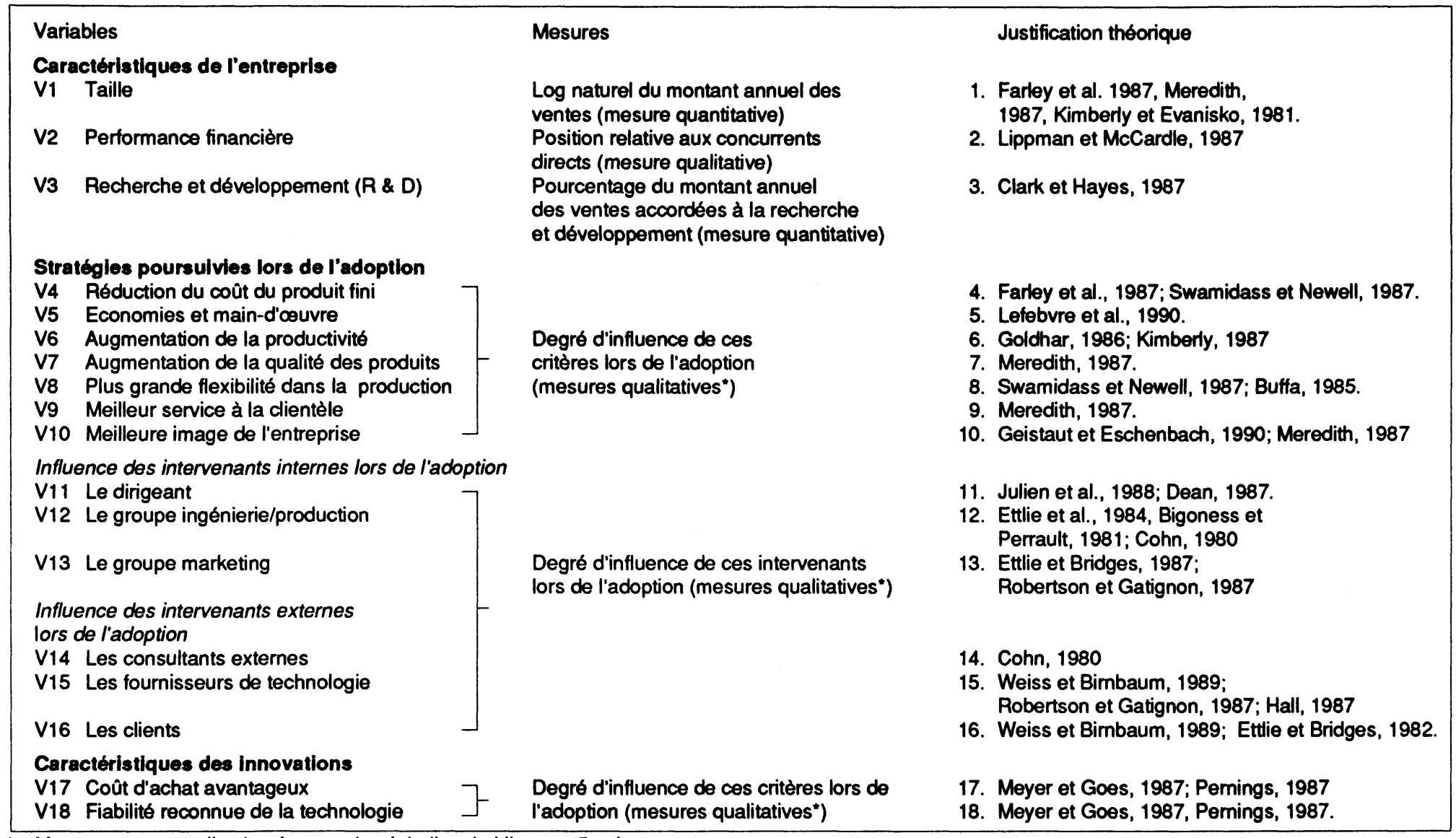

Mesures perceptuelles basées sur des échelles de Likert en 5 points. 
automatisés. Tous ces procédés de conception et de fabrication représentent des innovations où la technologie informatique est omniprésente. Au total, 144 entreprises ont répondu à ces conditions.

Afin d'atteindre notre premier objectif de recherche, une analyse factorielle a été effectuée dans le but de réduire le nombre de variables du modèle de départ à un nombre plus restreint de facteurs. La méthode de l'ACP (analyse en composantes principales) a été retenue pour assurer l'orthogonalité des facteurs, c'est-à-dire pour obtenir des facteurs non corrélés et ainsi permettre, dans un second temps, d'ordonner les facteurs selon deux groupes d'entreprises innovatrices : un premier groupe constitué d'entreprises ayant adopté une technologie avancée de production et un second groupe, composé d'entreprises plus innovatrices ayant adopté deux ou plusieursde ces technologies. Cesentreprises plusinnovatrices auraient non seulement franchi les premières phases de l'automatisation, mais elles auraient de plus réussi à intégrer un ensemble de technologies avancées de production dans leurs activités manufacturières.

\section{Résultats et discussion}

Les résultats de l'analyse factorielle menée sur l'ensemble des entreprises innovatrices nous permet de dégager six principaux facteurs qui expliquent $75 \%$ de la variance. Les six facteurs ainsi que la contribution des 18 variables de départ à chacun de ces facteurs sont présentés au tableau 1 (p. 222). Les faibles contributions enregistrées par chacune des variables s'expliquent par le nombre élevé des variables retenues (Lebart et al., 1977). Notons que lorsque les tailles d'échantillon sont relativement petites, les contributions supérieures à + ou -30 sont statistiquement significatives (Dillon et Goldstein, 1984).

Le premier facteur qui ressort est largement dominé par l'influence du groupe ingénierie-production lors de l'adoption. L'influence du groupe marketing est aussi présente mais dans une moindre importance. Il semblerait par ailleurs que la principale stratégie poursuivie lors de l'adoption des nouvelles technologies soit la flexibilité accrue. Ce facteur explique à lui seul $29 \%$ de la variance totale.

Le second facteur est associé à la troisième source d'influence interne de l'entreprise : l'influence du dirigeant. Ce facteur capte aussi une dimension stratégique, soit celle du meilleur service à la clientèle.

Le troisième facteur représente clairement les caractéristiques de l'entreprise; il est constitué de la taille et de la performance financière.

La dimension "coûts» domine le quatrième facteur. On y retrouve les économies de main-d'oeuvre comme principale stratégie poursuivie, et le coût avantageux de la technologie comme propriété majeure de l'innovation considérée. Bien sûr, l'adoption de technologies manufacturières de pointe représente un effort 
Tableau 1

Résultats de l'analyse factorielle' (avant rotation) ${ }^{2}$

\begin{tabular}{|c|c|c|c|c|c|c|c|c|}
\hline & & $\begin{array}{l}\text { Moyenne } \\
(n=144)\end{array}$ & $\begin{array}{c}\text { Factour } \\
1\end{array}$ & $\begin{array}{l}\text { Factour } \\
2\end{array}$ & $\begin{array}{l}\text { Facteur } \\
3\end{array}$ & $\begin{array}{c}\text { Factour } \\
4\end{array}$ & $\begin{array}{l}\text { Facteur } \\
5\end{array}$ & $\begin{array}{c}\text { Factour } \\
6\end{array}$ \\
\hline \multicolumn{9}{|c|}{ Caractóristiques do l'ontrepriso } \\
\hline $\mathbf{V}_{1}$ & Taille & 14.82 & 0.01 & -0.05 & 0.39 & -0.06 & -0.00 & 0.08 \\
\hline V2 & Performance financière & 3.73 & 0.10 & -0.04 & 0.36 & -0.05 & 0.05 & -0.03 \\
\hline V3 & Recherche et développement (R \& D) & 5.70 & 0.15 & 0.05 & -0.28 & -0.22 & 0.07 & 0.11 \\
\hline \multicolumn{9}{|c|}{ Stratbgies poursuivies lors do l'adoption } \\
\hline V4 & Réduction du coot du produn fini & 3.80 & 0.00 & 0.18 & -0.13 & 0.22 & 0.02 & -0.10 \\
\hline V5 & Economies et main-d'œuvre & 3.59 & -0.09 & -0.06 & 0.06 & 0.49 & 0.11 & -0.09 \\
\hline V6 & Augmentation de la productivité & 4.41 & 0.12 & 0.22 & -0.05 & 0.06 & -0.08 & -0.18 \\
\hline V7 & Augmentation de la qualité des produits & 4.03 & 0.12 & 0.04 & 0.04 & -0.04 & 0.01 & 0.16 \\
\hline V8 & Plus grande flexbilité dans la production & 3.80 & 0.32 & 0.06 & -0.02 & -0.09 & -0.12 & -0.07 \\
\hline V9 & Meilleur service à la clientèle & 4.33 & -0.06 & 0.35 & -0.04 & -0.07 & 0.04 & 0.05 \\
\hline V10 & Meilleure inage de l'entreprise & 3.63 & -0.15 & 0.02 & 0.06 & -0.01 & 0.00 & 0.50 \\
\hline \multicolumn{9}{|c|}{$\begin{array}{l}\text { Processus d'adoption } \\
\text { Influence des intervenants internes }\end{array}$} \\
\hline V11 & Le dirigeant & 4.43 & -0.22 & 0.51 & 0.01 & -0.20 & 0.06 & 0.01 \\
\hline V12 & Le groupe ingénierie/production & 3.73 & 0.43 & -0.27 & -0.02 & 0.15 & -0.11 & -0.20 \\
\hline V13 & Le groupe marketing & 3.18 & 0.30 & -0.02 & -0.02 & -0.26 & 0.13 & 0.09 \\
\hline \multicolumn{9}{|c|}{ Influence des intervenants externes } \\
\hline$V_{14}$ & Les consultants externes & 2.75 & 0.08 & 0.01 & 0.07 & -0.01 & 0.49 & 0.10 \\
\hline V15 & Les fournisseurs de technologie & 2.82 & 0.06 & 0.06 & -0.04 & 0.14 & 0.48 & -0.20 \\
\hline V16 & Les clients & 2.70 & 0.01 & -0.8 & -0.20 & -0.07 & -0.05 & 0.38 \\
\hline \multicolumn{9}{|c|}{ Caractéristiques des innovations } \\
\hline V17 & Coût d'achat avantageux de la technologie & 2.77 & -0.05 & -0.19 & -0.03 & 0.39 & -0.08 & 0.18 \\
\hline & Fiabilité reconnue de la technologie & 3.57 & 0.05 & 0.04 & 0.13 & 0.06 & 0.11 & 0.18 \\
\hline Valet & is propres & & 5.29 & 2.41 & 2.00 & 1.45 & 1.28 & 1.07 \\
\hline Pour & centage cumulatif de la variance expliquée & & $29 \%$ & $43 \%$ & $54 \%$ & $62 \%$ & $69 \%$ & $75 \%$ \\
\hline
\end{tabular}

Mesure de Kaiser-Meyer-Olkin' = 0,63 (mesure de validité de l'échantillon).

1 Toutes les variables sont mesurées à partir d'échelles de Likert de 1 à 5 (1 = influence très faible, 5 = influence très forte $)$ à l'exception de la taille exprimée sous forme de logarithme naturel des ventes, et de la recherche et développement sous formes de pourcentage des ventes consacrées au R \& D. Les mesures des variables étant différentes, toutes ont été standardisées lors de l'analyse factorielle.

2 Nous avons choisi de présenter les résultats sans rotation pour montrer les contributions initiales. 
de taille pour la majorité des PME. A cet égard, le coût avantageux de la technologie ne constitue qu'une dimension justificative du financement et la seule retenue pour cette recherche.

Le facteur suivant fait émerger l'influence des groupes externes à l'entreprise, en l'occurrence, celle des fournisseurs de technologie et celle des consultants externes.

Le dernier facteur est composé essentiellement de l'image que l'entreprise cherche à projeter en adoptant ces nouvelles technologies. On y retrouve, dans une moindre mesure, l'influence qu'exercent les clients de l'entreprise.

Tous ces facteurs représentent des dimensions relativement homogènes et complémentaires. Ainsi, à titre d'exemple, l'influence du groupe ingénierie-production observée dans le premier facteur est associée à la stratégie organisationnelle axée vers l'augmentation de la flexibilité dans les activités de production alors que dans le second facteur, l'influence du dirigeant est liéc à la dimension stratégique du meilleur service à la clientèle. Les regroupements observés suite aux résultats de l'analyse factorielle ne correspondent pas nécessairement aux regroupements imposés a priori puisque la composition des facteurs chevauche plusieurs dimensions. Chacun des facteurs peut alors être défini en fonction de la dominante qui s'en dégage :

\begin{tabular}{|c|c|c|c|}
\hline Facteur 1: & Influence interne & - & groupes fonctionnels \\
\hline Facteur 2: & Influence interne & - & direction \\
\hline Facteur 3 : & \multicolumn{3}{|c|}{ Influence des caractéristiques de l'entreprise } \\
\hline Facteur 4: & \multicolumn{3}{|c|}{ Influence des considérations de coûts } \\
\hline Facteur 5: & Influence externe & - & fournisseurs et consultants \\
\hline Facteur 6 : & Influence externe & - & clients et image de l'entreprise \\
\hline
\end{tabular}

Ces six facteurs sont ceux qui expliquent le mieux la dispersion observée dans un groupe d'entreprises qui ont toutes adopté des technologies avancées de production. Mais ces facteurs ont-ils un même degré d'importance relative selon que l'entreprise est plus ou moins engagée dans le processus d'innovation ? Pour répondre à cette question, les six facteurs ont été ordonnés selon la valeur de leurs moyennes (Manly, 1986). Le test de concordance de Kendall démontre que les deux groupes d'entreprises divergent totalement quant à l'importance accordée à chacun des facteurs (Tableau 2, p. 224).

Les résultats indiquent clairement que les entreprises plus innovatrices privilégient le sixième facteur qui est caractérisé par l'influence des clients etl'image de l'entreprise. Il semblerait donc que la PME manufacturière très innovatrice adopte les technologies avancées de production pour mieux répondre aux besoins spécifiques de ses clients. L'adoption de ces nouvelles technologies présente des atouts concurrentiels en permettant une plus grande diversité et spécificité des produits offerts (Meredith, 1987). Ces avantages correspondent à une stratégie de différenciation, stratégie traditionnellement préférée par les PME puisque qu'elles 
sont moins aptes à concurrencer par des stratégies de volume ou de coût (Lefebvre et al., 1990; Meredith, 1987).

\section{Tableau 2 \\ Ordonnance des facteurs' selon leur degré d'importance relative lors de l'adoption des technologies avancées de production}

\begin{tabular}{|c|c|c|c|}
\hline \multicolumn{4}{|c|}{ Ordre des facteurs ${ }^{2}$ dans : } \\
\hline & & $\begin{array}{l}\text { Entreprises } \\
\text { innovatrices }\end{array}$ & $\begin{array}{l}\text { Entreprises } \\
\text { très innovatrices }\end{array}$ \\
\hline Facteur 1: & Influence interne groupes fonctionnels & 5 & 2 \\
\hline Facteur 2: & Influence interne - direction & 3 & 4 \\
\hline Facteur 3: & Influence des caractéristiques de l'organisation & 1 & 6 \\
\hline Facteur 4: & Influence des considérations de coûts & 2 & 5 \\
\hline Facteur 5 : & Influence externe - fournisseurs et consultants & 4 & 3 \\
\hline Facteur 6: & Influence externe - clients et image de l'entreprise & 6 & 1 \\
\hline \multicolumn{4}{|c|}{$\begin{array}{l}\text { 1. Une analyse discriminante effectuée sur les deux groupes d'entreprises confirme ces } \\
\text { résultats puisque le facteur } 6 \text {, suivi du facteur } 1 \text {, discriminent le plus les deux groupes } \\
\text { d'entreprises (taux de classification obtenu par la méthode itérative (stepwise): } 64 \% \text { pour } \\
\text { le facteur } 6,72 \% \text { pour les facteurs } 6 \text { et } 1 \text { ). } \\
\text { 2. L'ordre des facteurs est complémentaire pour les deux groupes puisque l'analyse } \\
\text { factorielle standardise les variables ce qui a pour effet de porter à zéro la moyenne d'un } \\
\text { facteur. Bien que tous les facteurs soient importants puisqu'ils expliquent au total } 75 \% \\
\text { de la variance, cette analyse met en évidence le classement relatif des facteurs pour } \\
\text { chaque groupe. Le test de concordance de Kendall indique un désaccord total entre les } \\
\text { deux groupes : significatif à p } p=0.000 \text {. }\end{array}$} \\
\hline
\end{tabular}

L'image projetée par l'entreprise technologiquement avancée serait aussi une motivation importante lors de l'adoption. Selon les dirigeants de ces entreprises, cette projection externe de l'entreprise leur permet de se distinguer des concurrents. Ici encore, il pourrait s'agir d'une stratégie de différenciation basée sur le prestige associé à l'utilisation de technologies de pointe.

Le deuxième facteur par ordre d'importance est celui composé de l'influence des groupes fonctionnels au sein de ces entreprises. Il ressort en particulier que le groupe ingénierie-production joue un rôle dominant lors de l'adoption des technologies avancées de production dans les entreprises les plus innovatrices. Ce résultat concorde avec les résultats de recherches récentes qui ont souligné l'apport de la technocratisation dans l'adoption et la diffusion des innovations (Julien et al., 1988; Bigoness et Perreault, 1981; Cohn, 1980). Par ailleurs, il permet de nuancer l'importance du groupe ingénierie-production par rapport aux autres sources d'influence internes de l'entreprise y compris celle du groupe marketing. Cette influence va certainement de pair avec la stratégie manufacturière poursuivie, à savoir une plus 
grande flexibilité dans les activités de production. Ici encore, il s'agit d'un avantage concurrentiel qui soutient la stratégie de différenciation.

Au troisième rang, on retrouve l'influence des fournisseurs de technologie ainsi que celle des consultants externes, ce qui suggère que les entreprises plus innovatrices sont plus influencées par leur environnement externe, y compris l'environnement technologique (Robertson et Gatignon, 1987).

L'influence du dirigeant semble être similaire dans les deux groupes d'entreprises, ce qui dénote une certaine stabilité dans cette source d'influence, indépendamment du degré innovateur de l'entreprise. Par contre, les caractéristiques de l'entreprise et les considérations de coûts ne sont pas des facteurs dont l'influence serait déterminante dans les entreprises les plus innovatrices alors qu'ils sont primordiaux dans les entreprises moins innovatrices. Ceci suggère que l'influence de ces deux derniers facteurs est étroitement liée au degré d'innovation atteint et ce, malgré le fait que l'on considère uniquement des entreprises innovatrices. Ceci pourrait expliquer les résultats souvent contradictoires obtenus dans les recherches explorant la relation entre la taille et l'adoption d'innovations (Pennings, 1987). En effet, si la taille constitue un facteur important lors des premières phases d'automatisation d'une entreprise, elle serait nettement moins importante lors des phases subséquentes. Une relation comparable semble exister entre la performance financière et l'adoption ainsi qu'entre les considérations de coûts et l'adoption. En effet, nous soupçonnons que ces deux derniers facteurs ont un pouvoir discriminant beaucoup plus élevé pour dissocier les entreprises non automatisées des entreprises automatisées, et que ce pouvoir discriminant devient moindre lorsque les entreprises considérées sont automatisées à différents degrés. En d'autres termes, les facteurs d'adoption varieraient selon le degré d'automatisation atteint par une firme. Dans une perspective purement évolutive, tant les économistes (Amendola et Gaffard, 1988; Nelson et Winter, 1977) que les spécialistes en gestion des technologies (Nolan et Gibson, 1974) ont démontré l'effet cumulatif de l'expérience et de l'assimilation technologique dans la firme. Nous postulons ici que les facteurs incitant une entreprise à poursuivre ce processus technologique sont fortement liés au degré d'automatisation qu'elle a déjà atteint, ce qui correspond dans une certaine mesure à son vécu technologique.

Ainsi, la trajectoire technologique empruntée par une firme serait grandement influencée par un ensemble de conditions endogènes et exogènes qui favoriseraient non seulement l'assimilation mais également l'exploitation de cette technologie. Bien que la technologie peut en soi posséder certaines propriétés inhérentes qui permettent d'évaluer son utilité finale, il est essentiel de reconnaître que cette technologie est sujette à certains facteurs contextuels. Ces facteurs peuvent se situer au niveau de la firme, du secteur industriel ou de l'environnement économique et peuvent, en général, modérer ou même modifier les attentes à l'endroit de cette technologie. A cet égard, le processus définit la finalité même de la technologie. De plus, le processus est lui-même influencé par son origine puisqu'à divers degrés 
d'automatisation, l'accent mis sur les stratégies poursuivies ainsi que le degré d'influence des divers intervenants different.

\section{Conclusion}

Bien que l'adoption des nouvelles technologies avancées de production semble être l'apanage des grandes entreprises, un certain nombre de PME font figure de pionniers dans ce domaine. Ce rôle avant-gardiste en matière technologique comporte des risques qui peuvent engendrer des coûts substantiels pour l'entreprise. Par contre, l'adoption de ces technologies peut entraîner certains avantages concurrentiels. Cette ambivalence se retrouve aussi au niveau des déterminants d'adoption. Les résultats de cette recherche empirique menée auprès d'un échantillon de PME manufacturières innovatrices auront permis de reconnaitre six facteurs associés à l'adoption des technologies avancées de production. Ces six facteurs représentent différentes dimensions liées aux influences internes et externes de l'entreprise, aux caractéristiques de l'entreprise, aux considérations de coûts et aux stratégies poursuivies lors de l'adoption. Suite à l'analyse de l'importance relative de ces facteurs, il ressort que le classement varie selon le degré d'automatisation atteint. Dans l'entreprise plus innovatrice, identifiable par son degré d'automatisation plus élevé, les considérations d'ordre externe priment nettement sur les considérations financières. Il semblerait en effet que la stratégie poursuivie en est une de différenciation orientée sur l'image de l'entreprise et la reconnaissance des besoins de la clientèle. L'influence du groupe ingénierie-production combinée à la stratégie manufacturière axée vers une plus grande flexibilité dans les activités de production serait le deuxième facteur en ordre d'importance. Retenons toutefois que lorsque ces facteurs se retrouvent aussi dans les entreprises moins innovatrices, leur rôle est moins prédominant. L'étude des facteurs d'adoption doit donc tenir compte du degré d'automatisation des entreprises, ce qui suggère un modèle évolutif de l'adoption. Ainsi, certains des facteurs dominants lors des phases initiales d'automatisation tels que les facteurs «taille», «performance financière» et «coûts» seraient graduellement remplacés par d'autres facteurs moins axés sur des justifications structurelles et davantage sur des considérations de marché et de fonctionnement interne.

Compte tenu de ces résultats, l'étude d'un modèle évolutif pourrait s'avérer essentielle pour promouvoir l'adoption et la diffusion des nouvelles technologies dans les PME manufacturières.

\section{BIBLIOGRAPHIE}

Abernathy, W.J., Clark, K.B. et Kantrow, A.M., (1981), «The new industrial competition», Harvard Business Review, septembre-octobre, p. 68-81.

Amendola, M. et Gaffard, J.L., (1988), The Innovative Choice, Oxford, Basil Blackwell. 
Bergeron, F.et Buteau, C., (1988), «Devancer la concurrence par les systèmes d'information», Revue Internationale P.M.E., vol. 1, no 3-4, p. 295-307.

Bigoness, W.J. et Perreault Jr., W.D., (1981), «A conceptual paradigm and approach for the study of innovations", Academy of Management Journal, vol. 24, p. 68-82.

Buffa, E.S., (1985), «Meeting the competitive challenge with manufacturing strategy», National Productivity Review, printemps, p. 155-169.

Child, J., (1987), «Managerial strategies, new technology and the labour process» dans J.M. Pennings et A. Buitendam, (éds), New Technology as Organizational Innovation, Cambridge, Mass., Ballinger, p. 141-178.

Clark, K.B., Hayes, R.H. et Lorenz, C.,(1985), The Uneasy Alliance:Managing theProductivityTechnology Dilemna, Harvard Business School Press.

Clark, K.B. et Hayes, R.H., (1987), «Exploring factors affecting innovation and productivity growth within the business unit», dans K.B. Clark, R. H. Hayes et C. Lorenz (éds), The Uneasy Alliance: Managing the Productivity - Technology Dilemna, Boston, Mass, Ballinger, p. 35-58.

Cohn, S.F., (1980), «Characteristics of technically progressive firms», Omega, the International Journal of Management Science, vol. 8, no 4, p. 441-450.

Collins, P.D., Hage, J. et Hull, F.M., (1988), «Organizational and technological predictors of change in automaticity", Academy of Management Journal, vol. 31, no 3, p. 512-543.

CEC (Conseil économique du Canada), (1988), «Le tremplin commercial; une évaluation de l'accord canado-américain», catalogue EC22-151/1988f. Approvisionnement et services Canada, Ottawa.

Dean, J.W., (1987), «Building the future: the justification process for new technology», dans J.M. Pennings et A. Buitendam, (éds), New Technology as Organizational Innovation, Cambridge, Mass., Ballinger, pp. 35-58.

Dillon, W.R. et Goldstein, M., (1984), Multivariate Analysis: Methods and Applications, New York, John Wiley \& Sons.

Ettlie, J.E. et Bridges, W.P., (1982), «Environmental uncertainty and organizational technological policy», IEEE Transactions on Engineering Management, E.M. 29, no 1, février, p. 2-10.

Ettlie, J.E. et Bridges, W.P., (1987), «Technology policy and innovation in organizations», dans J.M. Pennings et A. Buitendam, (éds), New Technology as Organizational Innovation, Cambridge, Mass., Ballinger, p. 117-140.

Ettlie, J.E., Bridges, W.P. et O'Keefe, R.D., (1984), «Organization strategy and structural differences for radical versus incremental innovation», Management Science, vol. 30, no 6, juin, p. 682-65.

Farley, J.U.et al.,(1987), «Modeling the choice to automate», SloanManagement Review, vol. 29 , hiver, p. 5-15.

Gasse, Y., (1989), «A model of adoption process of new technologies for small and medium firms». Entrepreneurship: into the '90s, National Center for Management Research and Development, University of Westem of Ontario.

Geistaut, G.A. et Eschenbach, T.G., (1990), «Toward strategic propositions for technology management", dans, T.M. Khalil et B.A. Bayraktar (eds.), Management of technology, Norcross, Industrial Engineering and Management Press, p. 1177-1186.

Goldhar, J.D., (1986), «In the factory of the future, innovation is productivity», Research Management, mars-avril, p. 26-33.

Gomory, R.E., (1989), «From the "Ladder of Science" to the product development cycle», Harvard Business Review, vol. 67, novembre-décembre, p. 99-105. 
Hall, R.W., (1987), Attaining Manufacturing Excellence, Illinois, Dow Jones-Irwin, Homewood. Hayes, R.H.etWheelwright, S.C., (1984), Restoring our Competitive Edge: Competing Through Manufacturing, New York, John Wiley.

Hill, C.T., (1979), «Technological innovation: agent of growth and change», dans C.P. Hill et J.M. Utterback (éds), Technological Innovation for a Dynamic Economy.

Julien, P.A., Carrière, J.B. et Hébert, L., (1988), La diffusion des nouvelles technologies dans trois secteurs industriels, Conseil de la science et de la technologie, document no 88-03, avril, Québec.

Kamien, M.L. et Schwartz, N.L., (1982), Marketing Structure and Innovation, Cambridge, Cambridge University Press.

Kimberly,J.R.etEvanisko,M.J.,(1981), «Organizational innovation»,Academy of Management Journal, vol. 24, p. 689-713.

Kimberly, J.R., (1987), "Organizational and contextual influences on the diffusion of technolgical innovations», dans J.M. Pennings et A. Buitendam, (éds),New Technology as Organizational Innovation, Cambridge, Mass., Ballinger, p. 237-260.

Lebart, L. A., Morineau, A. et Fenelon, J.P., (1977), Traitement des données statistiques, Paris, Dunod.

Lefebvre, L.A. et Lefebvre, E., (1988), «Technologie et libre-échange - Une complicité souhaitable», L'Actualité économique. Revue d'analyse économique, vol. 64 , no 4, décembre, p. 616-629.

Lefebvre, L.A. etLefebvre, E., (1990), «Process innovators in the manufacturing sector», dans T.M. Khalil et B.A. Bayraktar (éds), Management of Technology, Industrial Engineering and Management Press, Norcross, p. 75-82.

Lefebvre, L.A., Lefebvre, E. et Colin, D., (1990), «Process innovation, productivity, and competitiveness", Revue canadienne des sciences administratives, (à paraitre).

Lippman, S.A. et McCardle, K.F., (1987), «Does cheaper, faster or better imply sooner in the timing of innovation decisions ?», Management Science, vol. 33, no 8, p. 1085-1064.

Litvak, I.A. et Warner, T.N., (1987), «Multinationals, advanced manufacturing technologies, and canadian public policy», Business Quarterly, été, p. 14-19.

Manly, B.F.J., (1986), Multivariate Statistical Methods: A Primer, New York, Chapman \& Hall.

McNeil, R.W. et Dilon, R.M., (1987), «Dealing with Canada's technology challenge by changing the corporate culture: the Ontario Research Foundation moves into the 1990"s.», Business Quarterly, automne, p. 40-44.

Meredith J., (1987), «The strategic advantages of new manufacturing technologies for small firms», Strategic Management Journal, vol. 8, p. 249-258.

Meyer, A.D. et Goes, J.B., (1987), «How organizations adopt and implement new technologies», Academy of Management Proceedings, p. 175-179.

Meyer, A.D. et Goes, J.B., (1988), «Organisational assimilation of innovations: a multilevel contextual analysis», Academy of Management Journal, vol. 31, no 4, p. 897-923.

Miller, D. et Toulouse, J.M., (1986), «Chief executive personality and corporate strategy and structure in small firms», Management Science, vol. 32, no 11, novembre, p. 1389-1409.

Naisbett, J. (1982), Megatrends, New York, Wamer Books.

Nelson, R.R. et Winter, S., (1977), «In search of a useful theory of innovation», Research Policy, vol. 6.

Nolan, R. et Gibson, C., (1974), «Managing the four stages of EDP growth», Harvard Business Review, vol. 52, janvier-février, p. 76-88. 
NRC (National Research Council), (1985), «Computer integrated manufacturing: barriers and opportunities», National Productivity Review, printemps, p. 170-177.

Parsons, G.L., (1983), «Information technology: a new competitive weapon», Sloan Management Review, vol. 25, automne, p. 2-14.

Pennings, J.M., (1987), «Technological innovations in manufacturing», dans J.M. Pennings et A. Buitendam (éds),NewTechnology as Organizational Innovation, ), Cambridge, Mass., Ballinger, p. 197-216.

Porter, M.E. et Millar, V.E., (1985), «How information gives you competitive advantage», Haward Business Review, vol. 63, juillet-août, p. 149-1660.

Quinn, J.B., Dooley, T.L., et Paquette, P.C., (1990), «Beyond products: services-based strategy", Harvard Business Review, vol. 68, mars-avril, p. 58-68.

Robertson T.S. et Gatignon, H., (1987), «The decision of high technology innovations: a marketing perspective, dans J.M. Pennings et A. Buitendam (éds), New Technology as Organizational Innovation, Cambridge, Mass., Ballinger, p. 179-196.

Rogers, E.M. et Eveland, J.D., (1975), «Diffusion: communication and innovation in organizations», dans P. Kelly et M. Kranzberg (éds),Aspects of Technological Innovation, Georgia Institute of Technology.

Skinner, W., (1985), Manufacturing: The Formidable Competitive Weapon, New York, John Wiley.

Swamidass, P.M.etNewell, W.T., (1987), «Manufacturing strategy, environmental uncertainty and performance: a path analytic model», Management Science, vol. 33, no 4, p. 509-524.

Thurow, L.C., (1987), «A weakness in process technology», Science, vol. 238, décembre, p. 1659-1663.

Weiss, A.R. et Birnbaum, P.H., (1989), «Technological infrastructure and the implementation of technological strategies», Management Science, vol. 35, n 8, p. 1014-1026. 